\title{
Surface acoustic waves in two dimensional phononic crystal with anisotropic inclusions
}

\author{
H. Ketata ${ }^{\text {a }}{ }^{1,2}$, M. Hédi Ben Ghozlen ${ }^{1}$ \\ ${ }^{1}$ Materials Physics Laboratory, Sfax University, Faculty of Sciences of Sfax, BP 11713000 Sfax \\ ${ }^{2}$ Preparatory Engineering Institute, Sfax University
}

\begin{abstract}
An analysis is given to the band structure of the two dimensional solid phononic crystal considered as a semi infinite medium. The lattice includes an array of elastic anisotropic materials with different shapes embedded in a uniform matrix. For illustration two kinds of phononic materials are assumed. A particular attention is devoted to the computational procedure which is mainly based on the plane wave expansion (PWE) method. It has been adapted to Matlab environment. Numerical calculations of the dispersion curves have been achieved by introducing particular functions which transform motion equations into an Eigen value problem. Significant improvements are obtained by increasing reasonably the number of Fourier components even when a large elastic mismatch is assumed. Such approach can be generalized to different types of symmetry and permit new physical properties as piezoelectricity to be added. The actual semi infinite phononic structure with a free surface has been shown to support surface acoustic waves (SAW). The obtained dispersion curves reveal band gaps in the SAW branches. It has been found that the influence, of the filling factor and anisotropy on their band gaps, is different from that of bulk waves.
\end{abstract}

Keywords. Phononic crystals, Surface acoustic wave, Band gap.

\section{Introduction}

The propagation of elastic or acoustic waves in periodic heterogeneous materials which is called phononic crystal (PCs) has received much attention in the last fifteen years $[1,2,3]$. One of the properties of composite materials is the possibility of having phononic band gaps, within which sound and vibrations at certain frequencies do not propagate. To probe the acoustic band structure of these 2D composites ultrasound transmission experiments in both the bulk and surface of the structures have been performed [4] These materials are of great interest for many applications, such as transducers, elastic/acoustic filters, noise control, and vibration shields. Most of previous works concentrates on PCs made of elastic isotropic materials; however, band gaps can be enlarged by using non-isotropic materials, such as piezoelectric materials [5]. At the micro-scale, phononic crystals are useful for acoustic isolation of vibrating structures [6]. Rigid substrate attachment improves yield, quality factor and immunity to environmental noise sources such as vibration.

To study the elastic wave behavior in this kind of system, several numerical analytical methods such as the plane wave expansion method (PWE) [7], the multi-scattering theory (MST) [8], and the finite-different time-domain method (FDTD) [9] have been developed and extensively used. Among

\footnotetext{
a e-mail : hassiba.ketata@yahoo.fr
} 
them, the PWE method, by which the wave equations are solved in the Fourier space. is mostly used to calculate the band structure [4].

The purpose of the present study is to elucidate theoretically and numerically the characteristics of bulk and surface acoustic waves in 2D phononic crystals. The presence of elastic anisotropy of the two chosen components materials should properly be taken into account. At low frequencies a complete band gap exists for same filling fraction and same mismatch in the elastic constants and mass densities of component materials.

The behavior of surface waves with respect to the filling fraction is different from a phononic material to another.

\section{Theory}

\section{1 Equations of wave motion}

We consider a two-dimensional phononic crystal shown in Fig. 1, which is infinite in $\mathrm{x}_{1}$ and $\mathrm{x}_{2}$ directions, with lattice constants a and coefficient of implantation $\mathrm{f}$.

The elastic wave propagating in elastic media can be described by these two equations:

$$
\begin{aligned}
& p \frac{a^{2} u_{i}}{a t^{2}}=\frac{g}{a x_{j}} \sigma_{i j}=\sigma_{i j i} \\
& \sigma_{i j}=c_{i j h} \frac{a u_{k}}{a x_{i}}=c_{i j h} u_{k i}
\end{aligned}
$$

where i, j, k, $1=1,2$ or $3, \boldsymbol{u}(\boldsymbol{r}, \boldsymbol{t})$ and $\sigma_{i j}(\boldsymbol{r}, \boldsymbol{t})$ represents the position and time dependant displacement vector and stress tensor respectively.

Considering the elastic isotropy of the studied materials, if we state propagation along $\mathrm{x}_{3}$ direction Eqs (1) and (2) can be simplified as

$$
\begin{aligned}
& \rho \omega^{2} u_{i}-\left(c_{n k 1} u_{k 1}+c_{i 1 k_{2}} u_{k, 2}\right)_{1}-\left(c_{n k 1} u_{k 1}+c_{k n 2} u_{k 2}\right)_{1}=
\end{aligned}
$$

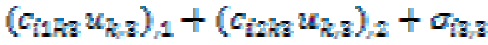

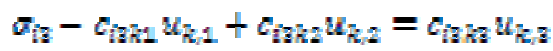

According to the periodicity of the structures, material constants $\alpha(\mathbf{r})$ (density $p(r)$ and rigidity tensor $c_{\text {ifil }}(\boldsymbol{r})$ can be expanded in the Fourier series

$p(r)=\Sigma_{G} \mathbb{R}^{Q} \exp (i G, r)$ and $G_{i j k i}(r)=\Sigma_{G} C_{i j i} \exp (i G, r)$

Based on the Bloch theorem, $\boldsymbol{u}(\boldsymbol{x}, \boldsymbol{t})$ and $\sigma_{i t}(\boldsymbol{r}, \boldsymbol{t})$ can be expanded in Fourier series:

$$
\begin{aligned}
& u(x, t)=e^{i\left(k_{0}, r_{12}-\omega t\right)} \Sigma_{G^{*}} e^{i G^{\prime} r_{12}} U^{G^{\prime}}(z) \\
& \sigma_{i}(x, t)=e^{i\left(R_{0}, r_{12}-u t\right)} \Sigma_{G^{*}} e^{i G^{\prime} r_{12} T_{i} g}(z)
\end{aligned}
$$

where $\omega$ is the wave circular frequency, $\mathbf{r}=x \varepsilon_{1}+y \varepsilon_{2}+z \varepsilon_{3}=r_{12}+z \varepsilon_{3}$ vector position, $e_{1}, e_{2}$ and $e_{3}$ being the unit vectors along the $\mathrm{x}_{1}, \mathrm{x}_{2}$ and $\mathrm{x}_{3}$ axes, $\boldsymbol{k}_{0}=\left(\boldsymbol{k}_{1}, k_{2}, 0\right)$ : the wave vector in the first irreducible Brillouin zone and $G($ or $G)=\left(n_{x} \frac{2 \pi}{a}, n_{y} \frac{2 \pi}{a}, 0\right)$, reciprocal lattice vectors where $\mathrm{n}_{\mathrm{x}}$ and $\mathrm{n}_{\mathrm{y}}=0, \pm 1, \pm 2, \ldots \ldots \pm \mathrm{M}$ if the summation over $\mathrm{G}$ is truncated to $\mathrm{n}$ values $\left(\mathrm{n}=(2 \mathrm{M}+1)^{2}\right)$

\subsection{Calculation of the band structure of bulk wave}

In the case where we study the dispersion curves of the bulk waves in the first Brillouin zone the displacement vector $\mathbf{u}$ tress tensor $\boldsymbol{\sigma}$ are independent of $\mathrm{z}$ then: 
$\sigma_{i 2,2}=0$ and $u_{n g}=0$

Substituting Eqs.(4) and (6) into Eq.(3), we can obtain the following generalized eigenvalue equation in the matrix form:

$$
\omega^{2}(R) U=(B) U
$$

Where $\boldsymbol{U}=\left[U_{1}^{Q^{5}}, U_{2}^{Q^{5}}, U_{s}^{G}\right]^{T}$ is the generalized Fourier amplitudes of displacement vector, (R) and (B) the $(3 n) \times(3 n)$ matrices which are functions of $\mathbf{k}_{\mathbf{0}}, \mathbf{G}, \mathbf{G}^{\prime}$ and the Fourier coefficients of material constants. The expressions of matrixes blocks $(3 \times 3)$ are:

$$
R_{i j}^{G G r}=R^{G-G r} \delta_{i j} \quad \text { and } B_{i R}^{G G r}=\sum_{j p} C_{i j h}^{G-g r}\left(k_{0}+G\right)_{j}\left(k_{0}+G^{h}\right)
$$

By solving equation (8) for $\omega$ as a function $\mathrm{n}$ of the wave vector $\mathbf{k}_{\mathbf{0}}$ in the first Brillouin zone, the band structures can be built.

\subsection{Surface waves}

In order to obtain the surface wave solution we further put

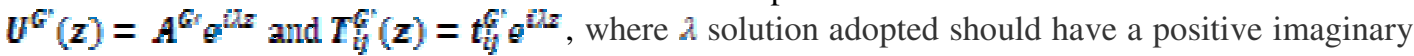
part

A plane wave expansion of Eqs (3) along $\mathrm{x}_{1}$ and $\mathrm{x}_{2}$ direction gives $3 \mathrm{n}$ equations for amplitude vectors $\left[A_{1}^{G^{*}}, A_{1}^{G}, A_{a}^{G}\right]^{T}$.

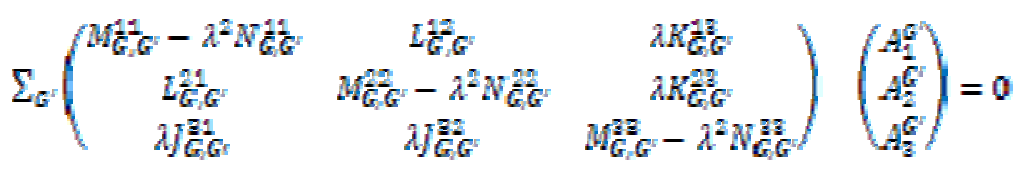

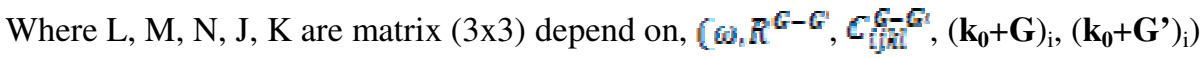

Putting, further, $A F^{r}=\lambda A^{r}$, If $(\mathrm{j}=1,2)$ and $A G^{r}=A g^{\prime}$, eq (9) is reduced to Eq (10)

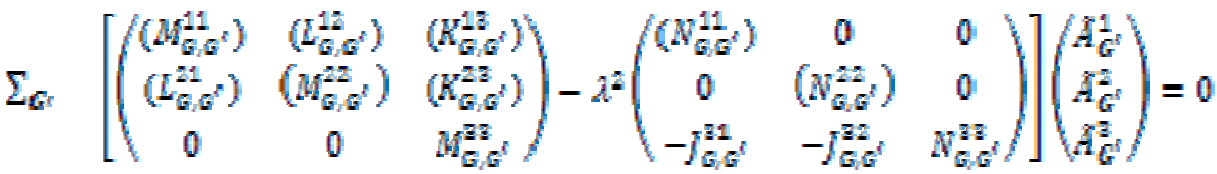

As well we obtain an generalized eigenvalue equation presented by equation (11) with respect to $\lambda^{2}$, which determines the spatial variation of the wave with the distance $\mathrm{z}$ from the surface,

$$
\left(Q_{G, G^{*}}-\lambda^{2} P_{G, G}\right) A_{G}=0
$$

$P, Q$ are $(3 n) x(3 n)$ matrix, if we truncate the expressions of Eqs (6) and (7) by choosing $n$ reciprocal lattice vectors. Equation (11) gives $3 n$ eigenvalues $\lambda_{2}^{2}(\varepsilon=1,2, \ldots 3 n)$.

For the surface wave we are seeking solutions for which the lattice displacement may decay exponentially into the medium ( $\mathrm{z}>0)$ away from the surface $\mathrm{z}=0$. If such a set of $\lambda_{\ell}$ 's are found for a given frequency $\boldsymbol{\omega}$ the displacement vector of the surface wave takes the form:

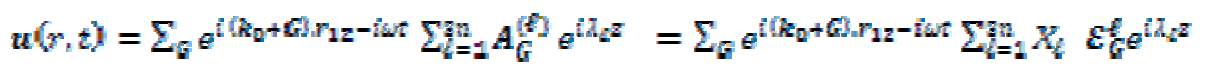

Where $\mathbb{E}$ is a unit polarization vectors and $X_{\mathscr{V}}$ the wave amplitudes.

The surface wave should satisfy the stress-free boundary condition at the surface $z=0$, whether: 
$\left.\sigma_{i}\right|_{z=0}=\left.c_{i \eta k l} \frac{\partial u_{k}}{\partial x_{f}}\right|_{z=0}=0 \quad(1=1,2,3)$

This leads to $3 \mathrm{n}$ homogeneous linear equations for $\boldsymbol{X}_{\boldsymbol{\ell}}$

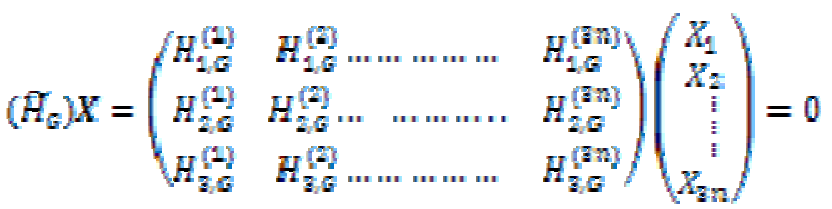

Where $\tilde{H}$ is $(3 n) \quad x \quad(3 n)$ matrix, and its elements are function of $\left(A_{i}, \varepsilon_{i G^{\prime}}^{G} C_{G-G^{i}}^{i j}\left(k_{0}+G\right)_{i}\left(k_{0}+G^{\prime}\right)_{i}\right)$,

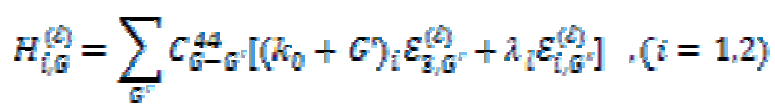

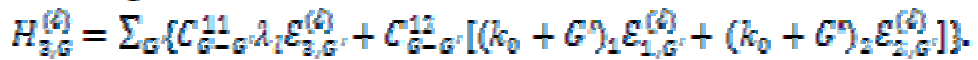

Equation (14) determines the relative weights $X_{/}$of $3 \mathrm{n}$ wave amplitudes if the frequency $\omega$ is correctly chosen. Actually we do not know a priori the eigenfrequency $\omega=\omega_{\mathbb{g}}$ of the surface localized acoustic mode, so Eq .(11) and the equation(16):

$$
\operatorname{det}(A)=0
$$

should be solved simultaneously to obtain $\omega_{z}$ and $\lambda_{l}$.

\section{Numerical Results}

We consider the structure where elastic square cylinders ( $\mathrm{W}$ or $\mathrm{Al}$ ) of section $\mathrm{s}=\mathrm{f} . \mathrm{a}^{2}$ are embedded periodically in a background material $\left(\mathrm{SiO}_{2}\right.$ or $\left.\mathrm{Si}\right)$, forming a square lattice with lattice spacing $\mathrm{a}=10^{-2} \mathrm{~m}(\mathrm{Fig}(1))$. The phononic materials studied in the present work are obtained from recent literature [6]. Materials are cubic crystals and elastic anisotropy is fully taken into account. Mechanical characteristics are presented in table 1 (annex). The irreducible part of the Brillouin zone is displayed in the figure (2).

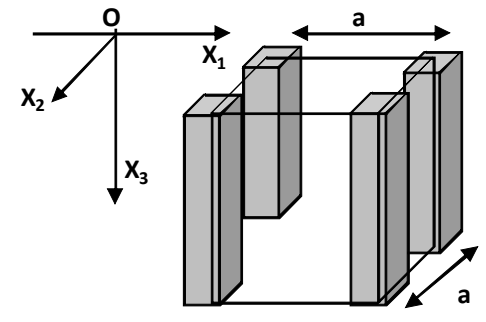

Fig.1.Schematic diagram of 2D square

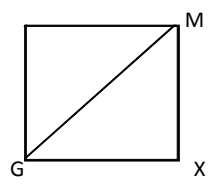

Fig. 2.Brillouin zone

\subsection{Dispersion diagram of bulk waves}

We have presented the dispersion curves of bulk waves along the boundary of the irreducible part of the Brillouin zone. Figures (3) shoes the case $\mathrm{W} / \mathrm{SiO}_{2}$ we note that for $\mathrm{f}=0.7$, (Fig (a)) there's no gap, while for $\mathrm{f}=0.25$, (Fig.(b)) an absolute gaps are present in three main directions of the Brillouin 
zone and this in the intervals of $\omega_{\mathrm{r}}\left(=\omega \mathrm{a} / \mathrm{v}_{\mathrm{t}}\right)$ [2.5-2.8] $(=$ gap1 $)$ and [3.6-3.8] $\left(=\right.$ gap2), where $\mathrm{a} / \mathrm{v}_{\mathrm{t}}=$ $2.65710^{-6} \mathrm{~s}$.

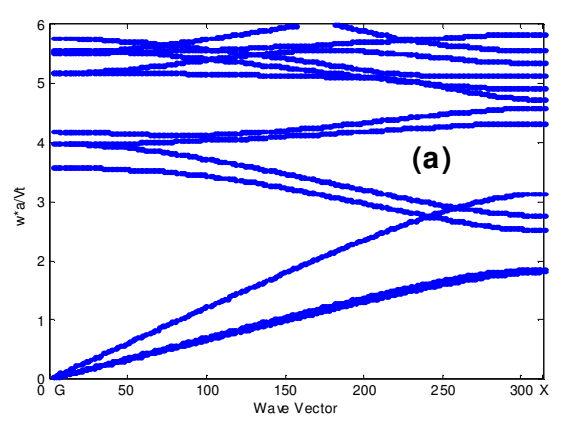

Fig.3.a. Dispersion curves of bulk acoustic waves in a two-dimensional square lattice consisting of $\mathrm{W} / \mathrm{SiO}_{2} . \mathrm{f}=0.7$

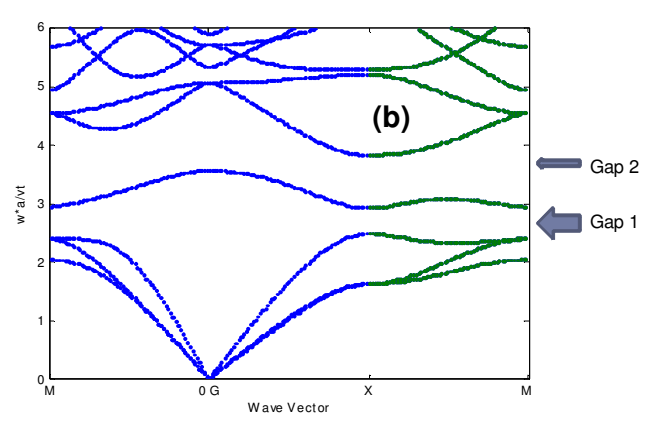

Fig.3.b.Dispersion curves of bulk acoustic waves $\mathrm{W} / \mathrm{SiO}_{2} . \mathrm{f}=0.25$

Figure 4 shows dispersion curves for $\mathrm{Al} / \mathrm{Si}$ with $\mathrm{f}=0.25$ but there's no gap, because those materials present a low density mismatch compared for $\mathrm{W} / \mathrm{SiO}_{2}$ (table 1)

Table 1. Mechanical characteristics of components phononic materials i:implantation, m:matrice

\begin{tabular}{|l|l|l|l|l|}
\hline Materiau & $\mathrm{Al}$ & $\mathrm{Si}$ & $\mathrm{W}$ & $\mathrm{SiO}_{2}$ \\
\hline$\rho\left(\mathrm{kg} / \mathrm{m}^{3}\right)$ & 2700 & 2329 & 19260 & 2203 \\
\hline $\mathrm{v}_{\mathrm{t}}(\mathrm{m} / \mathrm{s})$ & 3103.2 & 5842.5 & 2887.7 & 3763.3 \\
\hline $\mathrm{C}_{11}\left(10^{11} \mathrm{~Pa}\right)$ & 1.02 & 1.656 & 5.224 & 0.785 \\
\hline $\mathrm{C}_{12}\left(10^{11} \mathrm{~Pa}\right)$ & 0.50 & 0.639 & 2.044 & 0.161 \\
\hline $\mathrm{C}_{44}\left(10^{11} \mathrm{~Pa}\right)$ & 0.26 & 0.795 & 1.606 & 0.312 \\
\hline$\rho_{\mathrm{i}} / \rho_{\mathrm{m}}$ & 1.1593 & 8.7426 & \\
\hline $\mathrm{v}_{\mathrm{ti}} / \mathrm{v}_{\mathrm{tm}}$ & 0.53114 & 0.76732 & \\
\hline
\end{tabular}

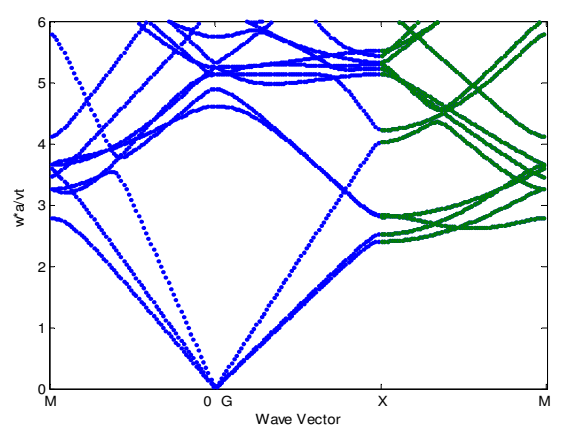

Fig.4. Dispersion curves of $\mathrm{Al} / \mathrm{Si}, \mathrm{f}=0.25, \mathrm{a} / \mathrm{vt}(\mathrm{Si})=1.7116 .10^{-6} \mathrm{~s}$

\subsection{Surface waves}

In figure 5 and 6 bulk and surface modes are presented in the direction of propagation GX. There are a few differences among transverse and surface modes. 


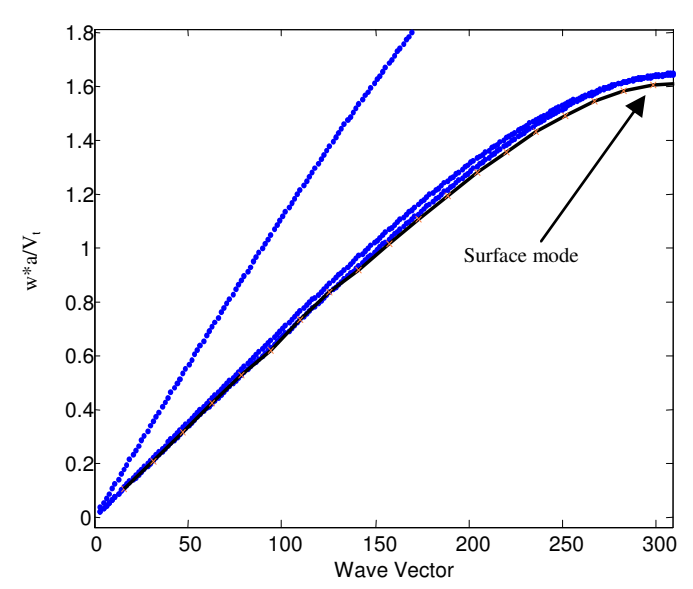

Fig.5. Reduced pulsation of bulk (-) and surface (--X--) modes at the GX direction of $\mathrm{W} / \mathrm{SiO}_{2}, \mathrm{f}=0.25$.

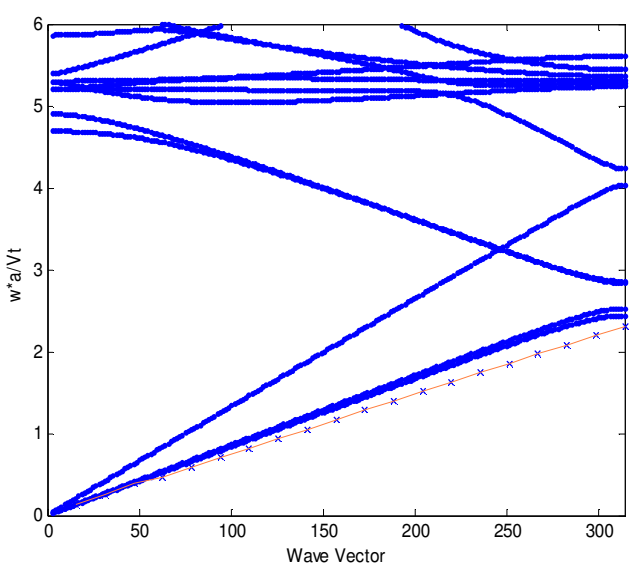

Fig. 6. Reduced pulsation of bulk $(-)$ and surface (--X--) modes(Ao) at the GX direction of $\mathrm{Al} / \mathrm{Si}, \mathrm{f}=0.25$.

As detailed in the previous paragraph the motion equation has been transformed in eigenvalue problem. The obtained solutions are divided into two sets, the lower and the upper frequency modes. When frequency is low the wavelength is comparatively large and the material behaves as an homogenous one. In addition the Rayleigh surface mode commonly called Ao is observed. For high frequency range several modes appear due to the complex nature of the phononic material.

Surface waves frequencies $\mathrm{w}_{\mathrm{s}}$ at the $\mathrm{X}$ point are plotted as the function of the filling fraction $\mathrm{f}$. In $\mathrm{W} / \mathrm{SiO}_{2}$ (figure 7) the both surface wave frequencies takes minimum value at $\mathrm{f}=0.35$. In $\mathrm{Al} / \mathrm{Si}$ (figure8) both surface waves decrease with $\mathrm{f}$. The investigated two cases $\mathrm{Al} / \mathrm{Si}$ and $\mathrm{W} / \mathrm{SiO}_{2}$ correspond to a low and high mismatch respectively. The mismatch is crucial to fix the number $\mathrm{n}$ of harmonics involved in the Fourier developments.

Additionally the largest gaps are associated with $\mathrm{W} / \mathrm{SiO}_{2}$ where the mismatch is extremely bigger [see Tab 1].

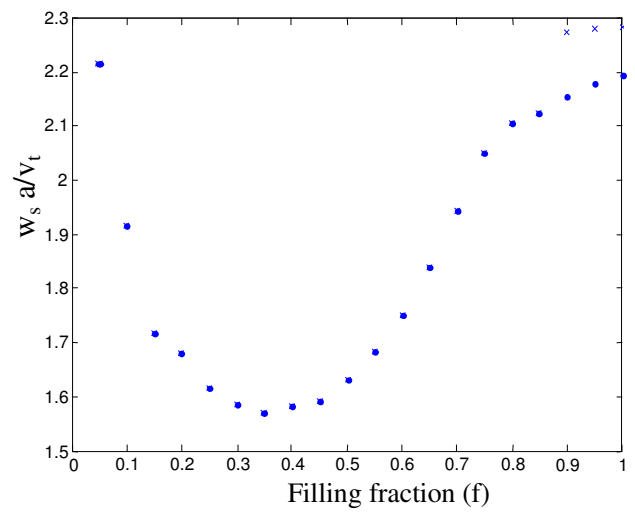

Fig.7. Frequency of the surface modes(x and $\cdot$ ) at the $\mathrm{X}$ point vs filling fraction (f) of $\mathrm{W} / \mathrm{SiO}_{2}$.

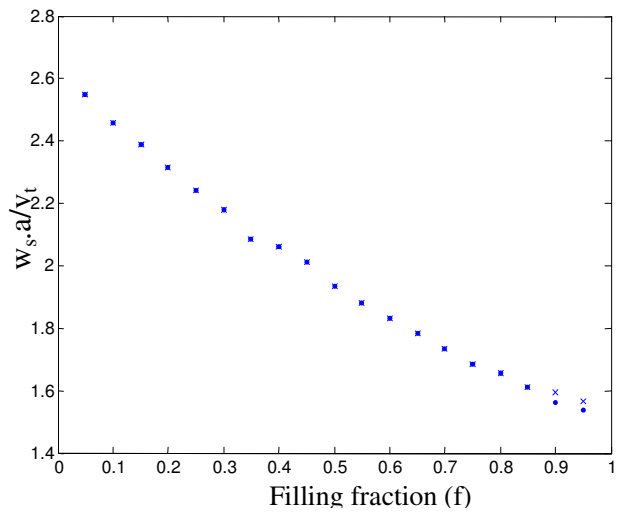

Fig.8. Reduced pulsation of the surface modes ( $\mathrm{x}$ and $\cdot$ ) at the $\mathrm{X}$ point vs filling fraction of $\mathrm{Al} / \mathrm{Si}$.

In figure 9 two branches of surface modes ( $\mathrm{ws}_{1}$ and $\mathrm{ws}_{2}$ ) and gap, that exist only for high value of filing fraction (fig 7) in $\mathrm{W} / \mathrm{SiO}_{2}$, are presented. By comparing our results with the results of Tanaka [2] who used the phononic materials AlAs/GaAs, we note that the behavior of phononic materials is very diverse. 


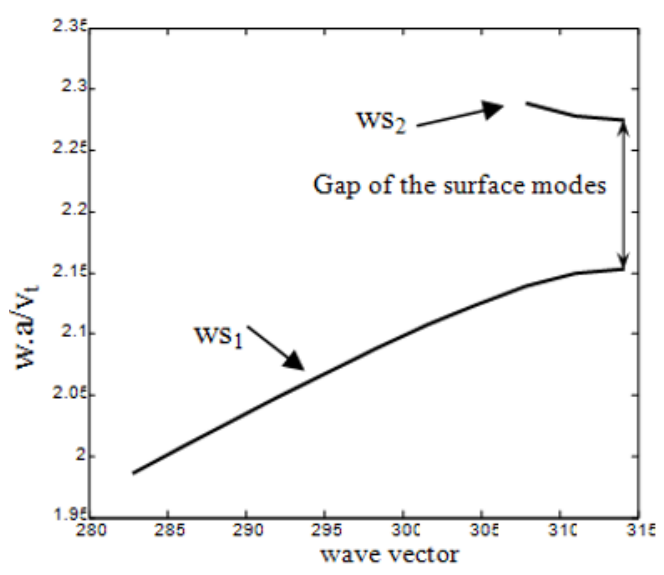

Fig.9. Reduced pulsation and gap of the surface modes at the $\mathrm{X}$ point, $\mathrm{f}=0.9$ of $\mathrm{W} / \mathrm{SiO}_{2}$

The dispersion curves, the surface waves, the gaps change each time we change the materials used, the geometry of the implantation, and essentially the ratio between the mechanical properties of both materials.

\section{Conclusion}

In the present work we have calculated the dispersion relations of bulk and surface acoustic waves in two-dimensional phononic crystals (PCs) consisting of periodic arrays of cylinders with square shapes embedded in a background substance. The acoustic band structures of 2D phononic crystals have been calculated with the plane-wave-expansion (PWE) method.

As examples, the band structures of $\mathrm{Al} / \mathrm{Si}$ and $\mathrm{W} / \mathrm{SiO}_{2}$ infinite and semi-infinite $\mathrm{PCs}$ are presented. The numerical calculation shows the acoustic band gaps between the dispersive curves result from large contrast in the elastic constants and mass densities of component materials. The calculation also demonstrate that the change in the frequency of the surface waves as a function of filling factor is not the same for the two compounds considered. For Al/Si frequency decreases when $\mathrm{f}$ increases, while for $\mathrm{W} / \mathrm{SiO}_{2}$ curve presents a minimum when $\mathrm{f}=3.5$. Finally surface waves gap is present only for high value of filing fraction in $\mathrm{W} / \mathrm{SiO}_{2}$.

\section{References}

1 M. S. Kushwaha, P. Halevi, Appl. Phys. lett. 641085 (1994)

2 Y. Tanaka, S.-I. Tamura, Phys. Rev. B 587958 (1998)

3 Xin Zhang, Youyan Liu, Fugen Wu, Zhengyou Liu Physics Letters A 314 144-149 (2003)

4 Yi-Ze Wang, Feng-Ming Li, Kikuo Kishimoto, Yue-Sheng, Wen-Hu Huang European Journal of Mechanics A/Solids 29 182-189 (2010)

5 F. R. Montero de Espinosa and al, Phys. Rev. Lett. 801208 (1998)

6 R. H. Olsson III, I. El-Kady Meas. Sci. Technol. 20012002 (2009)

7 Toshio Suziki and Paul K. L. Yu, J. Mech. Phys. Solids 46115 (1997).

8 Zhengyou Liu. C. T. Chan. Ping Sheng. A. L. Goertzen and J. H. Page. Phys. Rev. B 62. 2446 (2000)

9 J. O. Vasseur. P. A. Deymier. A. Khelif. Ph. Lambin. B. Djafari Rouhani, A. Akjouj, L. Dobrzynski, N. Fettouhi, and J. Zemmouri. Phys. Rev. E 65. 056608 (2002). 